\title{
Cancer Predisposition Sequencing Reporter (CPSR): A flexible variant report engine for high-throughput germline screening in cancer
}

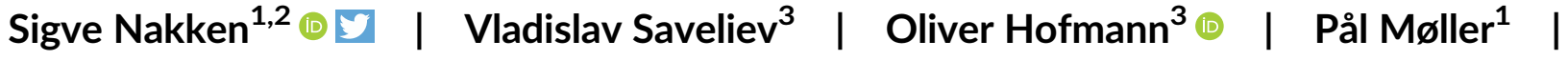 \\ Ola Myklebost ${ }^{1,4,5}$ (1) | Eivind Hovig ${ }^{1,6}$ (1)
}

${ }^{1}$ Department of Tumor Biology, Institute for Cancer Research, Oslo University Hospital, Oslo, Norway

${ }^{2}$ Centre for Cancer Cell Reprogramming, Institute of Clinical Medicine, Faculty of Medicine, University of Oslo, Oslo, Norway

${ }^{3}$ Centre for Cancer Research, University of Melbourne, Victoria, Australia

${ }^{4}$ Department of Clinical Science, University of Bergen, Bergen, Norway

${ }^{5}$ Western Norway Familial Cancer Center, Haukeland University Hospital, Bergen, Norway

${ }^{6}$ Centre for Bioinformatics, Department of Informatics, University of Oslo, Oslo, Norway

\section{Correspondence}

Sigve Nakken, Department of Tumor Biology, Institute for Cancer Research, Oslo University Hospital, Norway, and Centre for Cancer Cell Reprogramming, Institute of Clinical Medicine, Faculty of Medicine, University of Oslo, Norway.

Email: sigven@ifi.uio.no

Funding information

Norwegian Research Council, Grant/Award Number: 221580

\begin{abstract}
The value of high-throughput germline genetic testing is increasingly recognized in clinical cancer care. Disease-associated germline variants in cancer patients are important for risk management and surveillance, surgical decisions and can also have major implications for treatment strategies since many are in DNA repair genes. With the increasing availability of high-throughput DNA sequencing in cancer clinics and research, there is thus a need to provide clinically oriented sequencing reports for germline variants and their potential therapeutic relevance on a per-patient basis. To meet this need, we have developed the Cancer Predisposition Sequencing Reporter (CPSR), an open-source computational workflow that generates a structured report of germline variants identified in known cancer predisposition genes, highlighting markers of therapeutic, prognostic and diagnostic relevance. A fully automated variant classification procedure based on more than 30 refined American College of Medical Genetics and Genomics (ACMG) criteria represents an integral part of the workflow. Importantly, the set of cancer predisposition genes profiled in the report can be flexibly chosen from more than 40 virtual gene panels established by scientific experts, enabling customization of the report for different screening purposes and clinical contexts. The report can be configured to also list actionable secondary variant findings, as recommended by ACMG. CPSR demonstrates comparable sensitivity and specificity for the detection of pathogenic variants when compared to other algorithms in the field. Technically, the tool is implemented in Python/R, and is freely available through Docker technology. Source code, documentation, example reports and installation instructions are accessible via the project GitHub page: https:// github.com/sigven/cpsr.
\end{abstract}

\section{KEYWORDS}

bioinformatics workflow, cancer germline testing, clinical decision support, precision cancer medicine, variant interpretation

Abbreviations: ACMG, American College of Medical Genetics and Genomics; CIViC, clinical interpretation of variants in cancer; CPSR, Cancer Predisposition Sequencing Reporter; GWAS, genome-wide association studies; VCF, variant call format; VEP, variant effect predictor; VUS, variant of uncertain significance. 


\section{What's new?}

The Cancer Predisposition Sequencing Reporter is a unique bioinformatics tool for identifying genetic variants that may be therapeutically relevant. Many cancers arise from rare germline mutations in cancer predisposition genes. Knowing what variants a patient is carrying can help clinicians make decisions about risk-reduction interventions and surveillance, but interpretation of the variant profile can be challenging. This tool generates a report of variants in known cancer predisposition genes, highlighting those with therapeutic, prognostic, and diagnostic relevance.

\section{1 | INTRODUCTION}

A considerable fraction of human cancers is rooted in rare pathogenic germline mutations in cancer predisposition genes. ${ }^{1}$ Screening of cancer patients for predisposing germline alterations may yield valuable decision support for risk-reducing interventions and surveillance and has also proven its significance for the application of platinum-based chemotherapy and targeted drugs. ${ }^{2,3}$

High-throughput screening for a broad collection of cancer predisposition genes is currently feasible due to technological advances in genome-wide DNA sequencing. The accuracy of variant detection algorithms has improved substantially, producing consistent and highly accurate results, particularly for single point mutations. ${ }^{4}$ On the other hand, the ability to interpret variant findings in terms of clinical significance and actionability still represents a major challenge. To our knowledge, no freely available bioinformatics tool aims to transform raw germline variant sets to structured and interactive reports for clinical interpretation on a per-patient basis. Efforts in this area have focused primarily on the implementation of algorithms for variant pathogenicity classification, which lies at the core of clinical variant interpretation. Multiple tools and algorithms for variant classification according to published guidelines by the American College of Medical Genetics and Genomics (ACMG) have been developed, the most relevant ones in the field of cancer being CharGer, SherLoc, Cancer SIGVAR and PathoMAN ${ }^{5-8}$ (Supplementary Table 1). The comprehensive classification procedure outlined in Invitae's SherLoc framework is however not available as open-source software, and the limited webbased services offered by PathoMAN and Cancer SIGVAR are inconvenient for integration in high-throughput analysis environments. Furthermore, given the sensitive nature of DNA sequencing data from cancer patients, which is under strict regulations in most countries, it is frequently a necessity to choose stand-alone workflows over public web-based interpretation solutions. Also, neither of the abovementioned tools and algorithms provides structured genome reports on a case-by-case basis, and where the report content can be customized according to the cancer condition in question. Summing up, although the generation of informative variant interpretation reports constitutes an essential output of high-throughput cancer sequencing workflows, there is currently a shortage of flexible solutions for this in the open-source software landscape.

Here, we present a flexible bioinformatics tool that generates personal genome reports in the context of cancer predisposition and inherited cancer syndromes. Cancer Predisposition Sequencing
Reporter (CPSR) can be easily integrated with standard variant calling output from whole-genome, exome or targeted gene panel sequencing, and produces structured and interactive variant reports that highlight findings with clinical implications.

\section{2 | CONSTRUCTION AND CONTENT}

CPSR is implemented as a stand-alone bioinformatics workflow in Python and R. By design, it is therefore well suited for integration with workflows for high-throughput sequencing, as opposed to purely web-based solutions. Technically, CPSR builds upon our previously developed framework for the analysis of somatic mutations in tumor genomes, the Personal Cancer Genome Reporter. ${ }^{9}$ To facilitate reproducibility and ease of use, the tool can be installed either as a Dockerized application or through a Conda package, the latter probably being the preferred choice in high-performance computing environments. In addition to the actual software and configuration files, users need to download a dedicated data bundle, which contains the underlying databases that CPSR is using for functional variant annotation and as a basis for classification and reporting. CPSR supports both of the recent assembly versions of the human genome (ie, grch37 and grch38). Installation instructions and other information regarding configuration, versions of software and underlying databases, and input/ output files, are available from the project GitHub page (https:// github.com/sigven/cpsr), and also through the CPSR documentation website (https://cpsr.readthedocs.io).

The input to CPSR is a single file with DNA variants (SNVs/ InDels) detected from germline variant calling, encoded in the standard single-sample variant call format (VCF). CPSR automatically detects the genotype (homozygous/heterozygous) of input variants if these are formatted according to the correct standard in the VCF file. The workflow proceeds with four major steps, which are described in detail below (schematically illustrated in Figure 1).

\section{1 | Selection of targets for reporting-virtual cancer predisposition gene panels}

In order to serve a wide range of clinical cases, CPSR can produce variant reports that are dedicated towards predisposition genes for specific tumor types or cancer syndromes. In the initial step of the workflow, we exploit virtual gene panels as available from the 

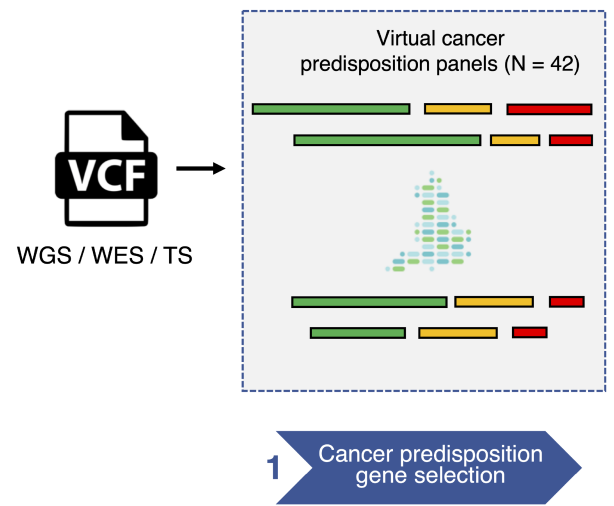
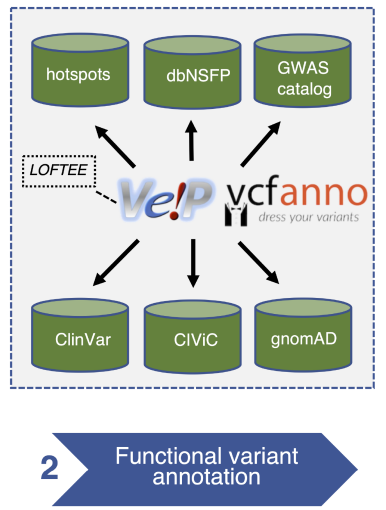
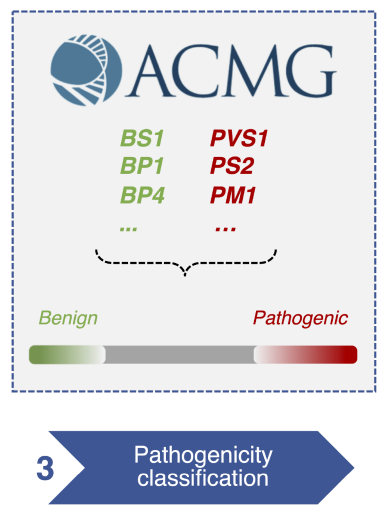
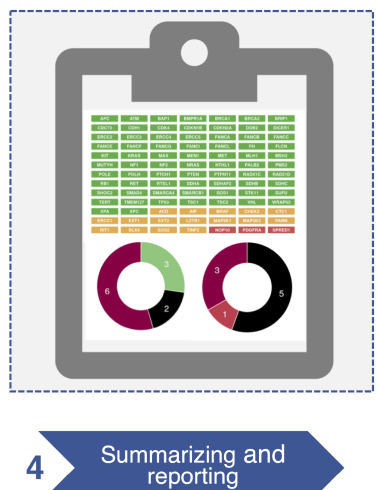

FIG URE 1 Cancer Predisposition Sequencing Reporter (CPSR) workflow with key databases and underlying software, illustrating how the query variant set from germline variant calling (formatted as VCF) is subject to four main steps for predisposition interpretation. Locus filtering against a selected cancer predisposition gene panel from the Genomics England PanelApp, where colors indicate confidence of association to phenotype, from diagnostic-grade in green to low-level confidence genes in red (step 1). Annotation through VEP and vcfanno with functional variant annotations: variant consequences by VEP, mutation hotspots from cancerhotspots.org, in silico deleteriousness predictions from dbNSFP, loss-of-function predictions through VEP's LOFTEE plugin, population allele frequencies from gnomAD, germline biomarkers from CIViC and low-risk alleles from NHGRI-EBI GWAS Catalog (step 2). Pathogenicity classification of novel variants according to a cancer-dedicated implementation of refined ACMG criteria (step 3). Aggregation and structuring of the results in a tiered cancer predisposition report (step 4). ACMG, American College of Medical Genetics and Genomics; CIViC, clinical interpretations of variants in cancer; dbNSFP, database of nonsynonymous functional predictions; gnomAD, genome aggregation database; LOFTEE, loss-of-function transcript effect estimator; TS, targeted sequencing; VCF, variant call format; VEP, variant effect predictor; WES, whole-exome sequencing; WGS, whole-genome sequencing

Genomics England PanelApp, a crowdsourcing initiative in which scientific experts are evaluating risk genes for more than 40 different hereditary cancer conditions on a continuous basis. ${ }^{10}$ Technically, variants in the input VCF file are filtered against the gene panel of choice (encoded as a BED file, both coding and noncoding regions) to ensure that variants analyzed are restricted to the panel genes only. When selecting a panel from PanelApp for analysis in CPSR, the user may also restrict the analysis to genes with a high level of disease association only (ie, diagnostic-grade or "GREEN" genes according to PanelApp nomenclature).

In addition to predefined panels from PanelApp, the user can choose to screen variants within a comprehensive exploratory panel intended for research use (ie, a "superpanel"), containing cancer predisposition genes gathered from multiple sources. As of June 2021 , the superpanel amounts to a total of 433 genes, containing all genes from PanelApp panels available in CPSR, genes curated in the Cancer Gene Census (COSMIC), those profiled in TCGA's PanCancer analysis of germline variants, and other user-contributed genes deemed relevant for cancer predisposition. Importantly, users may also flexibly define their own virtual screening panel from the set of genes in the exploratory superpanel.

Information on dominant vs recessive inheritance patterns for the various inherited cancer syndromes is largely harvested from the Genomics England PanelApp, with some additions from two other large-scale sequencing studies of cancer genes. ${ }^{1,11}$ Information related to the mechanism of disease (loss-of-function vs gain-of-function) per gene has been collected from the study by Maxwell et al. ${ }^{11}$ Disease-related gene properties are exploited during automated variant classification according to ACMG criteria (section Automated variant pathogenicity classification outlined below).

\subsection{Functional variant annotation}

The second step of the workflow utilizes two open-source tools, variant effect predictor (VEP) and vcfanno, to provide comprehensive functional annotations of all input variants. ${ }^{12,13}$

Gene variant consequences are determined by VEP, using GENCODE as the gene and transcript reference model. Cross-references to RefSeq transcripts are provided in the output whenever this is available through Ensembl's transcript database, which is important due to the frequent use of RefSeq identifiers in clinical variant reporting. Although a single variant frequently affects multiple transcripts in a given gene, CPSR reports a single main consequence per variant, using VEP's internal ranking routine to pick the most important transcript-specific consequence, a ranking that can be configured by the user (VEP options - pick_allele and -pick_order, see https:// www.ensembl.org/info/docs/tools/vep/script/vep_options.html\#basic). Notably, variants with a putative loss-of-function consequence (ie, stopgain, frameshift and splice site disruption), which are of major importance when it comes to pathogenic germline variants in cancer, are subject to careful evaluation and filtering through the loss-offunction transcript effect estimator (LOFTEE) plugin in VEP. ${ }^{14}$ Specifically, LOFTEE assigns confidence to a loss-of-function variant based on multiple features, such as transcript location, ancestral allele state and intron size and donor site nature (for splice site mutations). 
The relative location of variants with respect to intron-exon borders are also derived from VEP's output.

Through the use of vcfanno, the second workflow step will also annotate the input variants with data from multiple open-access variant datasets of relevance for cancer predisposition and functional variant effect (Figure 1). These datasets include information related to pre-classified variants in ClinVar (phenotypes, review status, etc.), population-specific allele frequencies (gnomAD, noncancer subset), known mutational hotspots in cancer (cancerhotspots.org), precomputed insilico deleteriousness predictions of missense and splice site variants (dbNSFP and dbSCSNV), low-risk risk alleles identified from genome-wide association studies of cancer phenotypes (GWAS catalog) and importantly, germline biomarkers of relevance for prognosis, diagnosis or therapeutic regimens retrieved from the clinical interpretation of variants in cancer resource (CIViC). ${ }^{14-20}$ Through annotations from $\mathrm{CIViC}$, we can effectively show which germline variants in the query that, according to published evidence from clinical trials or case reports, are likely to have therapeutic implications. A prominent example relates to cases with increased sensitivity to poly(ADP-ribose) polymerase (PARP) inhibitors elicited by pathogenic variants in BRCA1/2 genes, as well as other genes implicated in the homologous recombination repair pathway. ${ }^{21}$

\section{3 | Automated variant pathogenicity classification}

The occurrence of rare variants that have not yet received any classification or interpretation (ie, in ClinVar) is a common scenario in germline sequencing of cancer patients. To guide the interpretation of these variants, CPSR provides an automated pathogenicity classification in which the collection of variant annotations in step two (ie, consequence type, predicted functional effect and population frequencies), along with information on disease mechanism and mode of inheritance per cancer predisposition gene is exploited. Specifically, CPSR conducts a standard five-level (benign/likely benign [B/LB], variant of uncertain significance [VUS], likely pathogenic/pathogenic [LP/P]) variant pathogenicity classification, ${ }^{22}$ serving similar functionality to the open-source tools offered by CharGer and PathoMAN.

The classification procedure employed by CPSR is built largely upon the foundations established by the SherLoc algorithm, which made substantial refinements to the original ACMG guidelines for variant classification. ${ }^{7}$ In general, each ACMG criterion specifies particular properties of variants, such as population allele frequency and predicted functional effect that supports a pathogenic or benign variant nature. Furthermore, in the approach proposed by SherLoc, each ACMG criterion is weighted with positive or negative point scores that reflect their relative strength of importance with respect to classification. For all criteria that match with a given variant, scores are ultimately aggregated to obtain a single variant pathogenicity score. Notably, the specific combinations of software (eg, VEP and LOFTEE) and annotation databases (eg, GENCODE, gnomAD) used in CPSR, as well as the customization of the criteria towards the disease phenotype (eg, using cancer mutation hotspots to highlight important amino acids), are in effect providing a unique, cancer-dedicated variant classification procedure. The details of each ACMG evidence criterion implemented in CPSR, as well as their associated point scores, can be found in Supplementary Table 2.

Thresholds for converting variant pathogenicity scores to fivelevel classifications were calibrated through a comparison with existing ClinVar classifications (April 2021 release). In our calibration, we considered ClinVar variants in cancer predisposition genes (CPSR superpanel set v2.0, $n=335$ ), limited to variants with a review status of minimum two stars, the latter to minimize the impact of lowconfident variant interpretations (55 841 variants in total, see Supplementary Materials). The relationship between ClinVar classification status and variant pathogenicity scores calculated by CPSR is illustrated in Figure 2, and thresholds that were set ensured high concordance (agreement on $92.8 \%$ of all P/LP classified variants in ClinVar, 95.7\% for VUS variants and $96.3 \%$ for B/LB variants). The most challenging collection of variants with respect to correct score-based classification is located at the VUS/LP border, where the score is unable to discriminate accurately between VUS and LP/P variants. If such cases arise during analysis with CPSR, they are highlighted in the report, as a means to indicate that these should be carefully examined.

Finally, we compared the sensitivity and specificity of our classification algorithm with the two algorithms provided through CharGer (ie, custom and ACMG-based). Here, we used a benchmark variant set from the Pediatric Cancer Germline Project (PCGP) in which manual variant classifications defined by a panel of clinical geneticists constitute the gold standard (788 variants in total, see Supplementary Materials and Supplementary Table 3). For LP/P variants ( $n=105)$, classification with CPSR achieved a sensitivity of $74.3 \%$, which is higher than what was obtained with either of CharGer's two algorithms $(72.4 \%$ for the custom and $56.2 \%$ for the ACMG-based, respectively). Of all panel-determined nonpathogenic variants ( $n=683$ ), CPSR classified 16 variants as pathogenic, translating to a specificity of $97.7 \%$, an intermediate of the rates produced by CharGer's algorithms (98.1\% for the ACMG-based and $97.2 \%$ for the custom, respectively).

\section{4 | Variant report generation}

The final step of the workflow exploits the R Markdown framework to display all variant findings in a structured and interactive variant report. ${ }^{23}$ Of note is that additional output formats are also available to the user, that is, annotated VCF, JSON and TSV (tab-separated values). The TSV output can be utilized to collect results from multiple cases that have been analyzed with CPSR, which represents a common scenario in large research studies. An example HTML report can be downloaded for exploration here: https://doi.org/10.5281/ zenodo.5035949.

The interactive HTML report is organized into four main sections: Settings, Summary of Findings, Variant Classification and Documentation. 


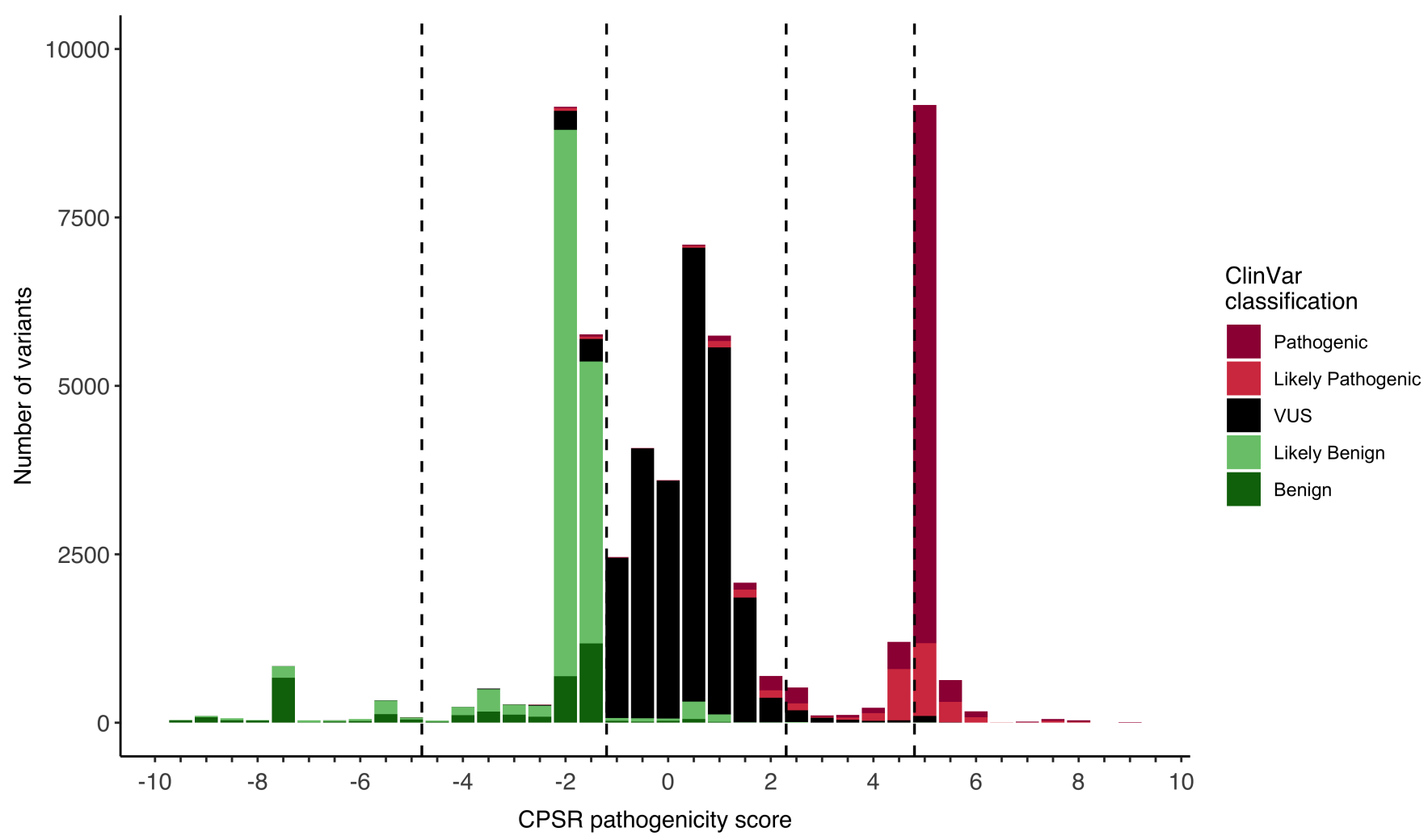

FIGURE 2 Calibration of Cancer Predisposition Sequencing Reporter (CPSR) pathogenicity score thresholds against ClinVar variants with a known classification (20210328 release, minimum two review stars). The complete distribution was calculated for variants in cancer predisposition genes $(n=335)$ and was used to determine suitable CPSR thresholds for P/LP/VUS/LB/B classifications, as indicated with the vertical dashed lines (pathogenic: [5,], likely pathogenic: [2.5, 4.5], VUS: [-1, 2.0], likely benign: $[-4.5,-1.5]$, benign: [, -5$]$ )

Settings indicate report and analysis configurations, as well as information regarding the virtual screening panel, while the Documentation section lists all versions of underlying databases and third-party software. These two sections thus serve to ensure reproducibility and transparency of the complete analysis workflow. Summary of findings provides the user with overall statistics with respect to classifications of variants found for the given case, both for variants already existing in ClinVar, and for novel variants without records in ClinVar.

The main content of the report is contained within the section named Variant Classification, where details of all variants are structured in interactive tables, and where the user can explore and filter variant data for various types of annotations, for example, population frequency, consequence type or existing phenotype associations. For variants of uncertain significance (VUS), which frequently make up the largest group of variants, the report importantly enables the use of the CPSR pathogenicity score to prioritize potential borderline cases. A dedicated biomarker section lists input variants that can have therapeutic implications or otherwise influence prognosis or diagnosis, and also allows the user to investigate the supporting literature and evidence for such associations.

Finally, the user can opt to list secondary pathogenic variants in a dedicated section, as recommended by $\mathrm{ACMG},{ }^{24}$ and also for a potential overlap of input variants with low-to-moderate risk alleles found for cancer phenotypes in GWAS.

\section{3 | DISCUSSION}

Knowledge on pathogenic variants in cancer-predisposing genes, and their relationships to systemic therapy choices, is emerging and evolving on a continuous basis. The quality and contents of the report produced with CPSR will thus advance accordingly, as underlying databases are updated. In particular, information regarding the mode of inheritance and the mechanism of action is currently not well established for a significant number of inherited cancers. Filling this gap is likely to improve variant classification in a number of genes.

The automated variant classification procedure implemented in CPSR demonstrated improved sensitivity over existing algorithms provided with CharGer. One should however note that simple comparisons of classification algorithms must be interpreted cautiously, primarily due to the fact that algorithms for variant classification are frequently configurable through a multitude of parameters. It should also be emphasized that automated procedures are intended primarily to guide the classification, and where borderline cases either way should be manually reviewed.

We acknowledge that additional datasets and analyses can add useful extra dimensions to the cancer predisposition report. A future version of CPSR should accept germline DNA copy number variants as an additional input type. Important pharma- and radiogenomic risk 
variants may further be incorporated during reporting, as well as a framework for calculation of polygenic risk scores. ${ }^{25}$

\section{4 | CONCLUSION}

Evidence-based personal cancer treatment based on genetic testing is an important goal in oncology. CPSR provides a documented tool to reach this goal.

\section{ACKNOWLEDGMENT}

This work was supported by grant 221580 from the Norwegian Research Council to the Norwegian Cancer Genomics Consortium.

\section{CONFLICT OF INTEREST}

The authors declared no potential conflicts of interest.

\section{DATA AVAILABILITY STATEMENT}

Source code and documentation of CPSR can be found through the project GitHub repository (https://github.com/sigven/cpsr). Supplementary materials, including supplementary tables, scripts and datasets for calibration/benchmark calculations are available here: https://doi.org/10.5281/zenodo.5035949. Further details and other data that support the findings of this study are available from the corresponding author upon request.

\section{ORCID}

Sigve Nakken (D) https://orcid.org/0000-0001-8468-2050

Oliver Hofmann (D) https://orcid.org/0000-0002-7738-1513

Ola Myklebost (D) https://orcid.org/0000-0002-2866-3223

Eivind Hovig (D) https://orcid.org/0000-0002-9103-1077

\section{TWITTER}

Sigve Nakken @ @igven

\section{REFERENCES}

1. Huang K-L, Mashl RJ, $\mathrm{Wu} Y$, et al. Pathogenic germline variants in 10,389 adult cancers. Cell. 2018;173:355-70.e14.

2. Thavaneswaran $\mathrm{S}$, Rath $\mathrm{E}$, Tucker $\mathrm{K}$, et al. Therapeutic implications of germline genetic findings in cancer. Nat Rev Clin Oncol. 2019;16:386-396.

3. Lincoln SE, Nussbaum RL, Kurian AW, et al. Yield and utility of germline testing following tumor sequencing in patients with cancer. JAMA Netw Open. 2020;3:e2019452.

4. Chen J, Li X, Zhong H, Meng Y, Du H. Systematic comparison of germline variant calling pipelines cross multiple next-generation sequencers. Sci Rep. 2019;9:9345.

5. Scott AD, Huang K-L, Weerasinghe A, et al. CharGer: clinical characterization of germline variants. Bioinformatics. 2019;35:865-867.

6. Ravichandran V, Shameer Z, Kemel Y, et al. Toward automation of germline variant curation in clinical cancer genetics. Genet Med. 2019; 21:2116-2125.

7. Nykamp K, Anderson M, Powers M, et al. Sherloc: a comprehensive refinement of the ACMG-AMP variant classification criteria. Genet Med. 2017;19:1105.

8. Li H, Liu S, Wang S, et al. Cancer SIGVAR: a semiautomated interpretation tool for germline variants of hereditary cancer-related genes. Hum Mutat. 2021;42:359-372.
9. Nakken S, Fournous G, Vodák D, Aasheim LB, Myklebost O, Hovig E. Personal cancer genome reporter: variant interpretation report for precision oncology. Bioinformatics. 2018;34: 1778-1780.

10. Martin AR, Williams E, Foulger RE, et al. PanelApp crowdsources expert knowledge to establish consensus diagnostic gene panels. Nat Genet. 2019;51:1560-1565.

11. Maxwell KN, Hart SN, Vijai J, et al. Evaluation of ACMG-guidelinebased variant classification of cancer susceptibility and non-cancerassociated genes in families affected by breast cancer. Am J Hum Genet. 2016;98:801-817.

12. McLaren W, Gil L, Hunt SE, et al. The Ensembl variant effect predictor. Genome Biol. 2016;17:122.

13. Pedersen BS, Layer RM, Quinlan AR. Vcfanno: fast, flexible annotation of genetic variants. Genome Biol. 2016;17:118.

14. Karczewski KJ, Francioli LC, Tiao G, et al. The mutational constraint spectrum quantified from variation in 141,456 humans. Nature. 2020; 581:434-443

15. Griffith M, Spies NC, Krysiak K, et al. CIViC is a community knowledgebase for expert crowdsourcing the clinical interpretation of variants in cancer. Nat Genet. 2017;49:170-174.

16. Liu X, Jian X, Boerwinkle E. dbNSFP: a lightweight database of human nonsynonymous SNPs and their functional predictions. Hum Mutat. 2011;32:894-899.

17. Landrum MJ, Lee JM, Riley GR, et al. ClinVar: public archive of relationships among sequence variation and human phenotype. Nucleic Acids Res. 2014;42:D980-D985.

18. Buniello A, MacArthur JAL, Cerezo M, et al. The NHGRI-EBI GWAS catalog of published genome-wide association studies, targeted arrays and summary statistics 2019. Nucleic Acids Res. 2019;47:D1005-D1012.

19. Chang MT, Bhattarai TS, Schram AM, et al. Accelerating discovery of functional mutant alleles in cancer. Cancer Discov. 2018;8:174-183.

20. Jian X, Boerwinkle $E$, Liu X. In silico prediction of splice-altering single nucleotide variants in the human genome. Nucleic Acids Res. 2014;42: 13534-13544.

21. Fong PC, Boss DS, Yap TA, et al. Inhibition of poly(ADP-ribose) polymerase in tumors from BRCA mutation carriers. N Engl J Med. 2009; 361:123-134.

22. Plon SE, Eccles DM, Easton D, et al. Sequence variant classification and reporting: recommendations for improving the interpretation of cancer susceptibility genetic test results. Hum Mutat. 2008;29:1282-1291.

23. Allaire JJ, Yihui Xie MJ, Javier L, et al. Rmarkdown: dynamic documents for R. 2019. https://github.com/rstudio/rmarkdown

24. Miller DT, Lee K, Chung WK, et al. ACMG SF v3.0 list for reporting of secondary findings in clinical exome and genome sequencing: a policy statement of the American College of Medical Genetics and Genomics (ACMG). Genet Med. 2021. https://doi.org/10.1038/s41436021-01172-3.

25. Torkamani A, Wineinger NE, Topol EJ. The personal and clinical utility of polygenic risk scores. Nat Rev Genet. 2018;19:581-590.

\section{SUPPORTING INFORMATION}

Additional supporting information may be found online in the Supporting Information section at the end of this article.

How to cite this article: Nakken S, Saveliev $\mathrm{V}$, Hofmann O, Møller P, Myklebost O, Hovig E. Cancer Predisposition Sequencing Reporter (CPSR): A flexible variant report engine for high-throughput germline screening in cancer. Int. J. Cancer. 2021;149(11):1955-1960. https://doi.org/10.1002/ijc. 33749 


\section{University Library}

\section{- M I N E R VA}

\section{A gateway to Melbourne's research publications}

Minerva Access is the Institutional Repository of The University of Melbourne

\section{Author/s:}

Nakken, S;Saveliev, V;Hofmann, O;Moller, P;Myklebost, O;Hovig, E

Title:

Cancer Predisposition Sequencing Reporter (CPSR): A flexible variant report engine for high-throughput germline screening in cancer

\section{Date:}

2021-08-04

\section{Citation:}

Nakken, S., Saveliev, V., Hofmann, O., Moller, P., Myklebost, O. \& Hovig, E. (2021). Cancer Predisposition Sequencing Reporter (CPSR): A flexible variant report engine for highthroughput germline screening in cancer. INTERNATIONAL JOURNAL OF CANCER, 149 (11), pp.1955-1960. https://doi.org/10.1002/ijc.33749.

Persistent Link:

http://hdl.handle.net/11343/289519

License:

CC BY-NC 